\title{
Motion Corrected 3D Reconstruction of the Fetal Thorax from Prenatal MRI
}

\author{
Bernhard Kainz ${ }^{1}$, Christina Malamateniou ${ }^{2}$, Maria Murgasova², Kevin \\ Keraudren $^{1}$, Mary Rutherford ${ }^{2}$, Joseph V. Hajnal ${ }^{2}$, and Daniel Rueckert ${ }^{1}$ \\ ${ }^{1}$ Imperial College London, Department of Computing, London, UK \\ \{b.kainz, kevin.keraudren10, d.rueckert\}@imperial.ac.uk \\ ${ }^{2}$ King's College London, Division of Imaging Sciences, London, UK \\ \{christina.malamateniou, maria.murgasova, mary.rutherford, jo.hajnal\} \\ @kcl.ac.uk
}

\begin{abstract}
In this paper we present a semi-automatic method for analysis of the fetal thorax in genuine three-dimensional volumes. After one initial click we localize the spine and accurately determine the volume of the fetal lung from high resolution volumetric images reconstructed from motion corrupted prenatal Magnetic Resonance Imaging (MRI). We compare the current state-of-the-art method of segmenting the lung in a slice-by-slice manner with the most recent multi-scan reconstruction methods. We use fast rotation invariant spherical harmonics image descriptors with Classification Forest ensemble learning methods to extract the spinal cord and show an efficient way to generate a segmentation prior for the fetal lung from this information for two different MRI field strengths. The spinal cord can be segmented with a DICE coefficient of 0.89 and the automatic lung segmentation has been evaluated with a DICE coefficient of 0.87 . We evaluate our method on 29 fetuses with a gestational age (GA) between 20 and 38 weeks and show that our computed segmentations and the manual ground truth correlate well with the recorded values in literature.
\end{abstract}

\section{Introduction}

Evaluation of the fetal thorax and especially fetal lung volumetry is an important diagnostic tool during prenatal screening and is considered to be an indicator for fetal health after birth. The respiratory function of the newborn highly depends on the overall alveolar surface, which in turn correlates with the lung volume [12]. To date, in the clinical practice, fetal lung volumetry is assessed only visually or by manually segmenting individual ultrasound slices. Usually only the lung main axes or a very rough manual segmentation is used to draw conclusions about fetal development [14]. Recently, fetal Magnetic Resonance Imaging (MRI) has been developed and allows to acquire high resolution slices from the fetus at a large field of view and with good tissue contrast [13]. However, the fetus is not sedated during these scans and may move freely inside the uterus. Because of a scan time of up to one second per slice, motion artifacts are likely to corrupt volumetric 
scans. Several orthogonal stacks of slices can be acquired and combined to one high resolution volume using approaches based on slice to volume registration (SVR). This way we obtain an artifact free, high resolution volume of a fetal target region $[11,15]$. So far, this process has been applied only to large, rigid and well structured fetal brain regions.

In this paper we aim to reconstruct high resolution 3D images of the fetal thorax and use them to automatically assess two key structures: lung and spine. Furthermore, we propose a method to evaluate these reconstructions fully automatically. The arbitrarily oriented spine is segmented using rotation invariant spherical harmonics image descriptors with a Classification Forest ensemble learning method. Lung tissue is subsequently identified by learning a graphical model of the distances between heart, left and right lung and the fetal main axis, which is defined by the orientation of the spinal cord.

Related Work: So far, fetal lung volumetry has been performed post mortem by weighing the inflated lungs in water and air [12] or in-vivo using either ultrasound [14] or manual segmentation of individual motion corrupted slices from MRI, taking only into account the thickness of the individual slices [9, 8]. An overview over the variability of these methods is given in [4]. Recently, significant advances for the automatic correction of fetal head movements and reconstruction to a high resolution volume have been achieved. Orthogonal stacks of misaligned 2D slices are processed using slice-to-volume registration and iterative super resolution, i.e., slices are registered to an improving estimation of the reconstructed 3D volume $[11,15]$. We have tested two publicly available frameworks with their default parameters and decided to adapt the framework in [11] for fetal thoracic reconstruction after a visual comparison of the results as shown in Fig. 1. This approach, using our datasets, shows clearer edges and less intensity inhomogeneities. Besides reconstruction methods, also semi-automatic and fully automatic approaches are available to identify fetal structures. So far, automatic localization and segmentation methods have only been applied to the fetal brain, using either multi stage localization via the eyes [1] or machine learning techniques applied to image feature descriptors $[6,10]$.

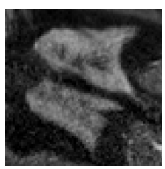

(a)

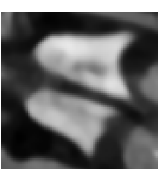

(b)

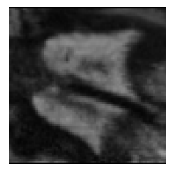

(c)

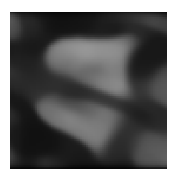

(d)

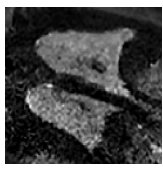

(e)

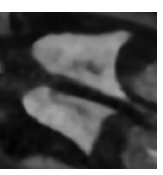

(f)

Fig. 1. Comparison of fetal thoracic super-resolution images with an isotropic reconstruction voxel size of $0.75 \mathrm{~mm}$. (a): originally acquired slice, (b): 10 iterations of edge preserving total variation (TV) minimizing denoising [16] of (a) in 3D, (c): reconstruction method proposed by [15], (d): TV denoising of (c), (e) reconstruction framework from [11], (f) TV denoising of (e). In this work we use a framework similar to [11] because it preserves more structure and has shown clearer edges during our experiments. We smooth and denoise the data after reconstruction using TV. 


\section{Method}

Overview: Our datasets are acquired on a Philips Achieva 1.5T (24 datasets) and $3 \mathrm{~T}$ scanner (5 datasets), the mother lying $20^{\circ}$ tilt on the left side to avoid pressure on the inferior vena cava. All scans have been ethically approved. A typical acquisition begins with a localizer scan, which is used to align the scan main axis approximately parallel to the fetus, and the selection of a region of interest. Single-shot fast spin echo (ssFSE) T2-weighted sequences are used to acquire a stack of images of the mother's womb. Each acquisition of a 2D image takes approximately $0.5-1.0 \mathrm{~s}$, which is fast enough to freeze fetal motion in each image, but generally results in inconsistent anatomical positioning between slices. The datasets show small to medium motion of the fetus. Several of these image stacks are acquired parallel and perpendicular to the fetus' main axis, i.e., axial, coronal and sagittal w.r.t. the fetus. The 3D resolution of each stack is approximately $288 \times 288 \times 90$ voxels with $1.2 \mathrm{~mm} \times 1.2 \mathrm{~mm} \times 1.25 \mathrm{~mm}$ voxel size for both used field strengths. An overview over our approach is given in Fig. 2. Our method requires a single click to define the center of the heart. Subsequently, we crop the motion corrupted stacks with a bounding box that is a third larger than expected for the size of the thorax at this age. In our experiments, this approach included the full fetal lung in all 2-6 stacks of images of the same subject.

Volume Reconstruction: It is possible to reconstruct a high resolution image $X$ from a number of motion corrupted stacks of $2 \mathrm{D}$ slice images $I_{i}, i \in 1 \ldots n$ using registration based super-resolution $[11,15]$. Therefore, the stacks are first rigidly registered to each other in $3 \mathrm{D}$ and during several motion correction iterations the individual slices are continuously rigidly registered to the current reconstruction of $X$ and reintegrated into $X$ using iterative gradient descent super-resolution with robust statistics to exclude mis-registered slices. Hence, the update equation for the super-resolution of a point $x_{i}$ in volume $X$ can be defined similar to [11] as $x_{i}^{(n+1)}=x_{i}^{n}+\alpha \sum_{j k} m_{i j}^{k} e_{j k}^{n}+\alpha \lambda \frac{\partial}{\partial x_{i}} R(X)$ (1), where $m_{i j}^{k} \in 1 \ldots N$ defines the relationship between acquired slices and the reconstructed volume as spatially aligned discretized Point Spread Function (PSF) for the acquisition of a voxel $y_{j k}$ from volume $X$ and $e_{j k}^{n}$ the error between the acquired slices and an intensity corrected simulated slice from the current reconstructed volume $X$. The regularization term $\alpha \lambda \frac{\partial}{\partial x_{i}} R(X)$ can be implemented as edge preserving smoothing following each super-resolution step. Robust statistics is implemented within an expectation maximization (EM) framework, which weights each voxel in a slice according to its registration quality and difference to it's simulated position within the reconstructed volume. To achieve a physical simulation of the image acquisition and a proper integration into $X$, slice voxels are modeled as discretized PSFs in [11], which can be approximated by a full width at half maximum Gaussian function for computational efficiency, scaled by the dimensions of the voxels [7].

While denoising of images is not very popular amongst physicians for diagnostics, we use TV minimizing denoising [16] to homogenize structures and to ease further post processing as shown in Fig. 1. 

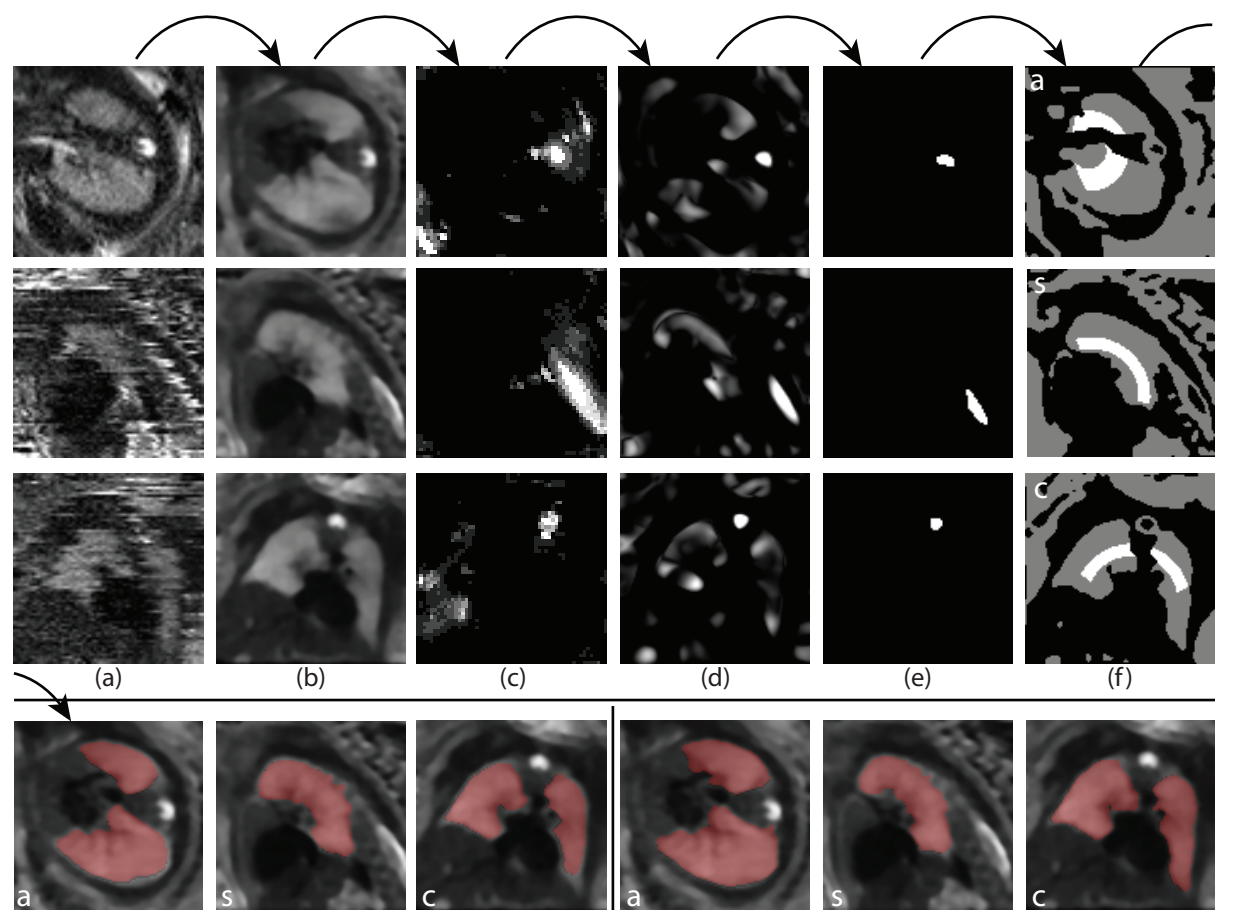

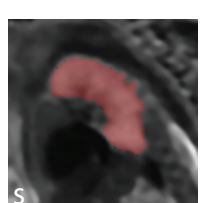

(g)

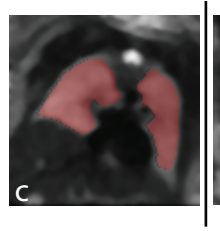

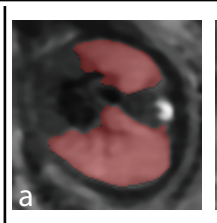

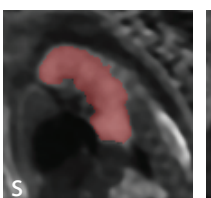

(h)

Fig. 2. An overview over our approach: From several motion corrupted image stacks (a) we reconstruct a high resolution volume (b) using Eq. 1, classify spinal cord voxels (c), Eq. 2, combine the spinal cord probability map with a tubular structure filter (d) and select the largest remaining region to determine the fetal main axis (e), Eq. 3, define foreground (white) and background (grey) constraints according to Eq. 4 in (f), and use them for a final segmentation utilizing constrained geodesic active contours [2]. (g) shows an example result from our approach and (h) shows the according manual $3 \mathrm{D}$ ground truth segmentation. (inside the images: a - axial, $\mathrm{s}-$ sagittal, $\mathrm{c}-$ coronal)

Spinal Cord Localization: The localization of any structure in the fetus is especially hard because of an unpredictable orientation of the fetus within the womb. To determine the orientation of the fetus, an automatic detection could try to register the scan to a known reference coordinate frame. This, however, is infeasible due to the influence of maternal tissues and the non-rigid nature of the fetus as a whole. A different approach is to derive image descriptors that are similar in any orientation of the image. Descriptors with such properties are called rotation invariant. Here, we propose a novel way to apply rotation invariant feature descriptors based on spherical harmonics to medical volume images from fetal MRI to localize the spine.

We define rotation invariant feature vectors with the angular power spectrum $\left\|a^{\prime l}\right\|^{2}$ of the expansion coefficients $a^{l}$ of spherical harmonics functions similar to [17]. For the expansion of the power spectrum we use spherical 3D Gabor basis 
functions, represented by a superposition of Bessel functions $\mathcal{B}_{s}^{l}(k)$. Therefore, our feature vector coefficients are at each voxel at an expansion $l$, scale $t$ and frequency $k: a_{l}^{k}(x)=\left(\frac{\sqrt{t}}{-k}\right)^{l} \bar{\nabla}^{l}\left(I \star \mathcal{B}_{s}^{0}(k)\right)(x)(2)$.

The transformation into the Gabor domain can be realized with one single initial cross correlation of the image and the basis function $I \star \mathcal{B}_{s}^{0}(k)$ followed by an iterative application of the spherical up-derivative operator $\bar{\nabla}^{l}$, which defines the spherical counterpart of a gradient operator in conventional calculus [17]. We found the optimal expansion bandwidth to be around 20 derivatives and obtained the best results with descriptor sizes between $6-11 \mathrm{~mm}$. We combine features at the minimum and the maximum of this range to form a feature vector consisting of coefficients for a smaller and a larger spherical neighborhood to gain additional descriptive power. Our final feature vector covers three different frequencies to represent the local image structure at each scale. We use $k=0, \pi$, and $2 \pi$. Consequently, each vector has a length of 63 elements per voxel.

We subsample the reconstructed volumes four times and learn every descriptor at every voxel position of a manually generated ground truth of the spinal cord from all high resolution reconstructions of our test datasets and use every third background descriptor for training a Classification Forest with 64 decision trees and a maximum depth of 128 [3]. The resulting probability map $P M_{s}(X)$ may show significant (non-tubular) outliers in regions where amniotic fluid and darker tissues form spine like patterns. Therefore, we filter the denoised volume using a 3D multi-scale vessel enhancement filter [5] for tubular structures between 3 to $6 \mathrm{~mm}$ and obtain an additional volume $\mathcal{V}(X)$ with a strong response for the spinal cord. However, this volume also shows responses in areas, where the fetus, the womb and the amniotic fluid form thick tube like structures. Robust detection with few false positives (false positive rate $<10 \%$ ) is achieved by a point-wise combination of the two detector methods, morphological opening and selection of the largest region of $S(X)=P M_{s}(X) \cdot \mathcal{V}(X)(3)$.

Lung Segmentation: The core of our method is the identification of lung tissue in $X$. Unfortunately, the method described for spine localization cannot be applied directly to the lungs because of its more complex and partitioned shape. Because we know already the center position of the heart and the voxels of the spinal cord we can generate a probability map for lung voxels based on a graphical model of the thoracic organs. We use logistic regression to learn and predict the gestational age (GA) specific distances between the center of the heart $c_{H}$, and the centers of the left $c_{L u l}$ and the right lung $c_{L u r}$ from our datasets. The learned distances are the length of the vectors $\boldsymbol{v} \mathbf{1}=\left(c_{L u l}-c_{H}\right), \boldsymbol{v} \mathbf{2}=\left(c_{L u r}-c_{H}\right)$, $\boldsymbol{v} \mathbf{3}=\left(c_{\text {Lul }}-c_{\text {Lur }}\right)$, hence $d_{H}^{L u}=(\|\boldsymbol{v} \mathbf{1}\|+\|\boldsymbol{v} \mathbf{2}\|) / 2$ (we assume symmetry) and $d_{\text {Lul }}^{\text {Lur }}=\|\boldsymbol{v} \mathbf{3}\|$. Typical values for the linear prediction model $a \cdot x+b$ from our test datasets can be found in Fig. 3 (for specific gestational age (GA) the exact parameters are: $d_{H}^{L u}=0.145 \cdot G A-5.966$ and $\left.d_{L u l}^{L u r}=0.1747 \cdot G A-1.141\right)$. Furthermore, we use the spinal cord segmentation to define a line $s$ through the main axis of the spine using RANSAC and the normal vector $\boldsymbol{n}_{H}^{s}$ to this line going through $c_{H}$. All voxels, which are in an expected lung intensity range $\left[l i_{1}, l i_{2}\right]$, manually measured from the ground truth segmentation independently 
for $1.5 \mathrm{~T}$ and $3 \mathrm{~T}$ data, and within the age specific predicted distances $d_{L u l}^{L u r}$ and $d_{H}^{L u}$ and in between $c_{H}$ and $s$ are subsequently defined as conservative estimation of lung voxels. All voxels that have been identified as neither belonging to a tubular structure nor likely to be a spine voxel nor being within the expected lung intensity range, hence $S(X) \equiv 0$, are defined to be background. Therefore, we obtain a trinary volume for lung foreground 1 , background -1 , and uncertain regions 0 for all voxels $\left\{v_{x y z} \in X \mid l i_{1} \leq v_{x y z} \leq l i_{2}\right\}$ and vectors $\boldsymbol{u}=v_{x y z}-c_{H}$,

$$
\mathcal{U}(X)= \begin{cases}1 & \text { if } S(X) \equiv 0 \wedge-\boldsymbol{n}_{H}^{s} \cdot \boldsymbol{u}>\alpha \wedge \\ & \left(d_{H}^{L u}-\left(d_{H}^{L u} * \gamma\right) \leq\|\boldsymbol{u}\| \leq\left(d_{H}^{L u}+\left(d_{H}^{L u} * \gamma\right)\right.\right. \\ -1 \quad & \text { if } S(X) \neq 0 \vee l i_{1} \geq v_{x y z} \geq l i_{2} \\ 0 & \text { else. }\end{cases}
$$

$\alpha=0.5$ and $\gamma=0.1$ revealed to be good choices during our experiments. Subsequently, we use $\mathcal{U}(X)$ as initial foreground/background constraints for our implementation of geodesic active contours segmentation [2]. Finally, the lung volume $V$ results for all $N$ classified lung voxels by $V=\frac{\sum_{i=0}^{N} s^{3}}{1000}[m l]$, with $s$ as the reconstructed isotropic voxel size in $\mathrm{mm}(0.75 \mathrm{~mm}$ during our experiments).

\section{Evaluation and Results}

Ground truth segmentations for the reconstructed datasets have been generated in 3D using a few voxels wide manual brush strokes to find larger homogeneous regions quickly with geodesic active contours segmentation [2]. This initial segmentation is subsequently manually refined by an expert using one voxel wide foreground and background constraints. As a baseline we have also generated manual slice-by-slice segmentations of the lungs from a single stack per dataset similar to the current state of the art [8]. Fig. 3 compares the exponential growth regression curves of post mortem measurements, slice-by-slice evaluation with multiplication by the slice thickness from literature, our slice-by-slice ground truth, the ground truth acquired from manual 3D segmentation of the reconstructions and the results from our semi-automatic approach.

Our method has been evaluated on an Intel Xeon E5-2643 system with 16 GB RAM and a Nvidia Titan Graphics card (GPU). The reconstruction to a high resolution volume takes $\sim 10 \pm 5$ min - depending on the number of input stacks and the size of the region of interest - with our own GPU accelerated (CUDA) implementation of [11]. This is $\sim 25 \times$ faster than the original CPU implementation. The transformation to feature space (GPU) and the voxel classification (CPU) takes on average $3.4 s$. The segmentation refinement (CPU) takes $15 s$. Therefore, our approach can be executed within approximately 15 minutes.

We used 10-fold cross-validation for the spinal cord localization training and generated a manual ground truth of the spine similar to the 3D lung ground truth. On average the automatic spinal cord segmentation reached a DICE coefficient of $0.89\left(\sigma^{2}=0.001\right)$ compared to the manual ground truth during our experiments. The automatic segmentation of the lung volume achieves a similarly high DICE coefficient of $\mathbf{0 . 8 7}\left(\sigma^{2}=0.002\right)$. 


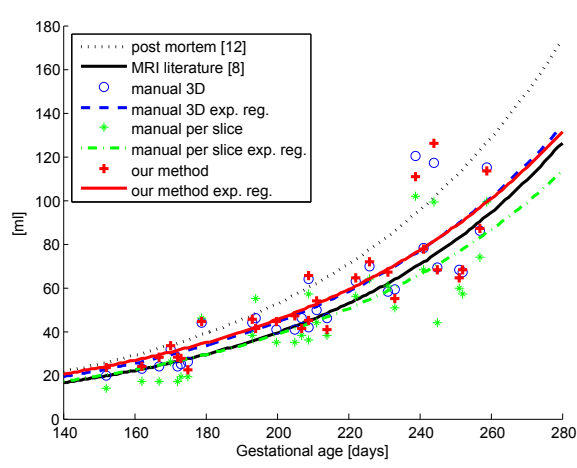

(a)

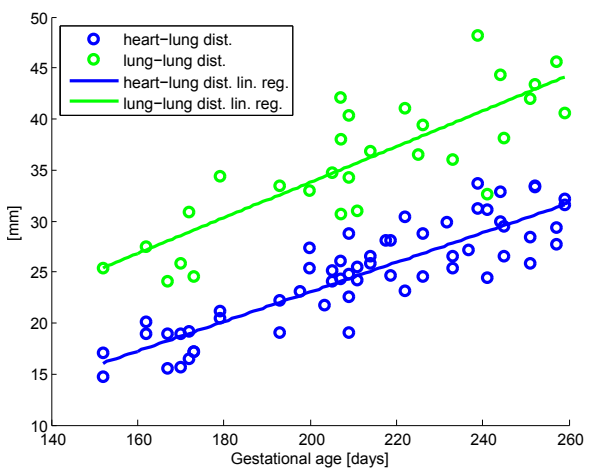

(b)

Fig. 3. (a) compares the results from post mortem measurements [12] (dotted, black), slice wise measurements from MRI literature [8] (line, black), our manual segmentation from 3D reconstructions (blue o) with its exponential regression curve (blue dashed line), manual slice wise segmentation (green $*$ ) with its exponential regression curve (green dashed line), and our automatic method (red + ) with its its exponential regression curve (red line). (b) shows the learned distances between the selected heart center point and the center of the lungs. (exp. = exponential, lin. = linear, reg. = regression)

\section{Discussion and Conclusion}

We have shown motion corrected reconstruction and almost fully automatic analysis of the fetal thorax in a genuine volumetric approach. Our method works well for cases of small to medium motion. If all scanned input stacks are corrupted by strong motion we have observed that the results for the lung volume become more similar to the manual slice-by-slice approach. We have shown that the evaluated methods produce measurements within the clinically known natural variation of the fetal growth. While the state-of-the-art approach requires time consuming slice-by-slice segmentation, our method requires only a single click, which can be done when the radiographer defines the scan region. Our results show that when performing lung volumetry on motion corrected high resolution reconstructions, the values appear to be closer to post mortem measurements.

With minor changes (different definition of the expected lung intensity) the presented approach is applicable to two different MRI field strengths and reliably localizes healthy lung tissues in the fetal thorax. The higher signal-to-noise ratio of $3 \mathrm{~T}$ input is likely to improve our results if only $3 \mathrm{~T}$ datasets are used for training. We will evaluate the segmentation performance w.r.t. the field strength in future work as soon as we have acquired more 3T data. The localization of the fetal spine and the resulting automatic estimation of the fetal main axis can also be used as basis for further diagnostics and automated image analysis.

Acknowledgements: Bernhard Kainz is supported by an EU Marie Curie Intra-European Fellowship (FP7-PEOPLE-2012-IEF F.A.U.S.T. 325661). 


\section{References}

1. Anquez, J., Angelini, E., Bloch, I.: Automatic Segmentation of Head Structures on Fetal MRI. In: IEEE ISBI'09. pp. 109-112. IEEE Press, New York (2009)

2. Criminisi, A., Sharp, T., Blake, A.: GeoS: Geodesic Image Segmentation. In: Forsyth, D.A., Torr, P.H.S., Zisserman, A. (eds.) ECCV'08, Part I. LNCS, vol. 5302, pp. 99-112. Springer Berlin Heidelberg (2008)

3. Criminisi, A., Shotton, J.: Decision Forests for Computer Vision and Medical Image Analysis. Springer-Verlag New York Inc. (2013)

4. Deshmukh, S., Rubesova, E., Barth, R.: MR Assessment of Normal Fetal Lung Volumes: A Literature Review. AJR Am J Roentgenol 194(2), W212-7 (2010)

5. Frangi, A.F., Niessen, W.J., Vincken, K.L., Viergever, M.A.: Multiscale Vessel Enhancement Filtering. In: Wells, W.M., Colchester, A., Delp, S. (eds.) MICCAI'98, LNCS, vol. 1496, pp. 130-137. Springer Berlin Heidelberg (1998)

6. Ison, M., Donner, R., Dittrich, E., Kasprian, G., Prayer, D., Langs, G.: Fully Automated Brain Extraction and Orientation in Raw Fetal MRI. In: Proc. Workshop on Paediatric and Perinatal Imaging, MICCAI'12. pp. 17-24 (2012)

7. Jiang, S., Xue, H., Glover, A., Rutherford, M., Rueckert, D., Hajnal, J.V.: MRI of Moving Subjects Using Multislice Snapshot Images with Volume Reconstruction (SVR): Application to Fetal, Neonatal, and Adult Brain Studies. IEEE T Med Imaging 26(7), 967-980 (Jul 2007)

8. Kasprian, G., Balassy, C., Brugger, P., Prayer, D.: MRI of Normal and Pathological Fetal Lung Development. Eur J Radiol 57(2), 261-70 (2006)

9. Keller, T.M., Rake, A., Michel, S.C.A., Seifert, B., Wisser, J., Marincek, B., KubikHuch, R.A.: MR Assessment of Fetal Lung Development Using Lung Volumes and Signal Intensities. Eur Radiol 14(6), 984-9 (2004)

10. Keraudren, K., Kyriakopoulou, V., Rutherford, M.A., Hajnal, J.V., Rueckert, D. Localisation of the Brain in Fetal MRI Using Bundled SIFT Features. In: Mori, K., Sakuma, I., Sato, Y., Barillot, C., Navab, N. (eds.) MICCAI'13, Part I. LNCS, vol. 8149, pp. 582-589. Springer Berlin Heidelberg (2013)

11. Kuklisova-Murgasova, M., Quaghebeur, G., Rutherford, M.A., Hajnal, J.V., Schnabel, J.A.: Reconstruction of Fetal Brain MRI with Intensity Matching and Complete Outlier Removal. Medical Image Analysis 16(8), 1550-60 (2012)

12. Langston, C., Kida, K., Reed, M., Thurlbeck, W.M.: Human Lung Growth in Late Gestation and in the Neonate. Am Rev Respir Dis 129(4), 607-613 (Apr 1984)

13. Levine, D.: Fetal Magnetic Resonance Imaging. J Matern Fetal Neonatal Med 15(2), 85-94 (Feb 2004)

14. Moeglin, D., Talmant, C., Duyme, M., Lopez, A.C.: Fetal Lung Volumetry Using Two- and Three-Dimensional Ultrasound. Ultrasound in Obstetrics and Gynecology 25(2), 119-127 (2005)

15. Rousseau, F., Oubel, E., Pontabry, J., Schweitzer, M., Studholme, C., Koob, M., Dietemann, J.L.: BTK: An Open-Source Toolkit for Fetal Brain MR Image Processing. Computer Methods and Programs in Biomedicine 109(1), 65-73 (2013)

16. Rudin, L.I., Osher, S., Fatemi, E.: Nonlinear Total Variation Based Noise Removal Algorithms. Physica D: Nonlinear Phenomena 60(14), 259-268 (1992)

17. Skibbe, H., Reisert, M., Schmidt, T., Brox, T., Ronneberger, O., Burkhardt, H.: Fast Rotation Invariant 3D Feature Computation Utilizing Efficient Local Neighborhood Operators. IEEE Trans. Pat. Anal. Mach. Intell. 34(8), 1563-1575 (2012) 